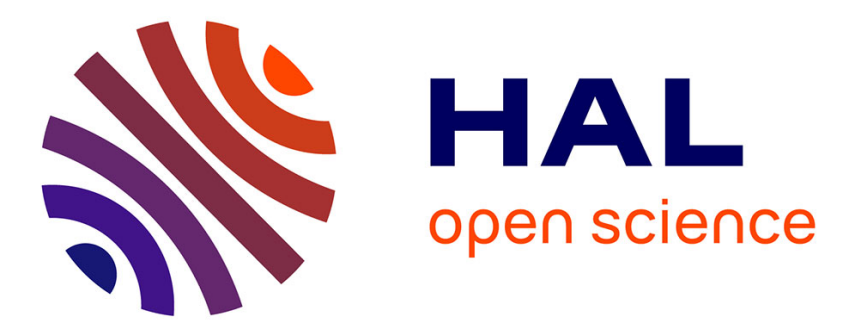

\title{
Exciton magnetic polarons in semimagnetic quantum wells and superlattices \\ D. Yakovlev
}

\section{To cite this version:}

D. Yakovlev. Exciton magnetic polarons in semimagnetic quantum wells and superlattices. Journal de Physique IV Proceedings, 1993, 03 (C5), pp.C5-67-C5-74. 10.1051/jp4:1993512 . jpa-00251598

\section{HAL Id: jpa-00251598 https://hal.science/jpa-00251598}

Submitted on 1 Jan 1993

HAL is a multi-disciplinary open access archive for the deposit and dissemination of scientific research documents, whether they are published or not. The documents may come from teaching and research institutions in France or abroad, or from public or private research centers.
L'archive ouverte pluridisciplinaire HAL, est destinée au dépôt et à la diffusion de documents scientifiques de niveau recherche, publiés ou non, émanant des établissements d'enseignement et de recherche français ou étrangers, des laboratoires publics ou privés. 


\title{
Exciton magnetic polarons in semimagnetic quantum wells and superlattices
}

\author{
D.R. YAKOVLEV
}

Physikalisches Institut der Universität Würzburg, 97074 Würzburg, Germany

and I.F. Ioffe Physico-Technical Institute, Russian Academy of Sciences, 194021 St. Petersburg, Russia

\begin{abstract}
We review the experimental and theoretical investigations of static and dynamical properties of magnetic polarons in $\mathrm{CdTe} /(\mathrm{Cd}, \mathrm{Mn}) \mathrm{Te}$ quantum wells and superlattices. Magnetic polarons are formed from excitons primarily localized on the fluctuations of quantum well width (or fluctuations of superlattice periods). The polaron energy has been measured as a function of structure parameters, temperature and external magnetic fields applied parallel and perpendicular to the growth axis. Dynamics of the magnetic polaron formation has been studied by use of picosecond time-resolved luminescence. A theory of magnetic polarons in quantum wells developed in the adiabatic approximation gives good qualitative description of the experimental results. A strong effect of the spin-glass phase modification at interfaces on the magnetic polaron energies in quantum wells and superlattices has been found.
\end{abstract}

\section{Introduction}

The strong exchange interaction between spins of carriers and magnetic ions in semimagnetic semiconductors causes their unique properties including giant Zeeman splitting of the band states, large Faraday rotation, and magnetic polarons [1]. Localized carriers or excitons may ferromagnetically organize the spins of magnetic ions within their Bohr orbits via exchange interaction and form magnetic polarons [2]. A type of primary localization of excitons is of key importance for the polaron formation process. Magnetic polarons formed from carriers or excitons bound to impurities have been studied intensively and are known as bound magnetic polarons [2]. A wavefunction of the ground state of the bound magnetic polarons is controlled by the electrostatic potential of the impurity center. For localized exciton magnetic polarons the formation process is accompanied by the autolocalization and squeezing of the wavefunctions. So far, there are only few studies of localized exciton magnetic polarons in bulk semimagnetic semiconductors $[3,4,5]$.

The recent technological progress in molecular-beam epitaxy growth of the heterostructures with semimagnetic layers made possible the investigation of magnetic polarons in quantum wells and superlattices. The formation of exciton magnetic polarons has been demonstrated in $\mathrm{CdTe} / \mathrm{Cd}_{1-\mathrm{x}} \mathrm{Mn}_{\mathrm{x}} \mathrm{Te}$ single quantum well (SQW) structures [6]. In this paper we present the review of our studies of the exciton magnetic polaron phenomenon in $\mathrm{CdTe} / \mathrm{Cd}_{1-\mathrm{x}} \mathrm{Mn}_{\mathrm{x}} \mathrm{Te}$ quantum well structures. Static properties of magnetic polarons and the dynamics of polaron formation are discussed. We compare experimental data with the results of variational calculations developed in the adiabatic approximation [7,8]. The effect of the spin-glass phase modification at interfaces on the magnetic polaron energy is demonstrated. Finally we present recent results on the exciton magnetic polaron formation in short-period superlattices. 

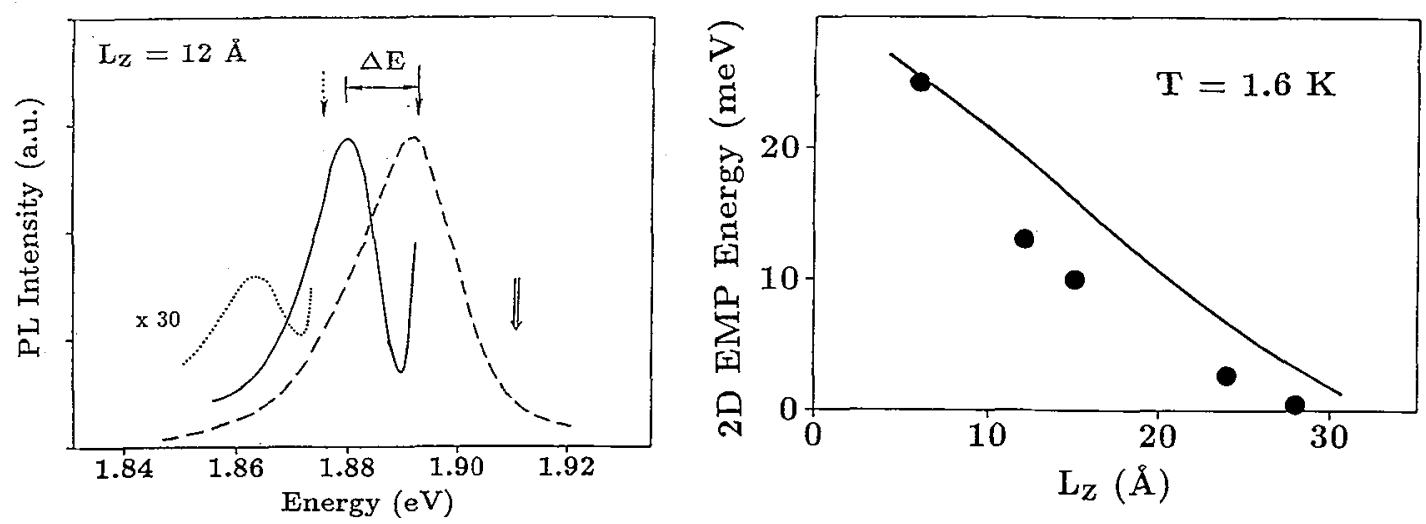

Fig.1 Photoluminescence spectra of a CdTe $/ \mathrm{Cd}_{0.73} \mathrm{Mn}_{0.27} \mathrm{Te} \mathrm{SQW}$ with $L_{z}=12 \AA$ at $T=1.6 \mathrm{~K}$. Energy of the free heavy-hole excitons $n=1$ is shown by an open arrow. Spectra under nonresonant excitation $\hbar \omega_{\text {exc }}=$ $1.95 \mathrm{eV}$ (dashed line) and under selective excitation in the localized exciton states with $\hbar \omega_{\text {exc }}=1.892 \mathrm{eV}$ (solid) and $\hbar \omega_{\text {exc }}=1.875 \mathrm{eV}$ (dotted) are shown [6]. Arrows indicate the selective excitation energies.

Fig.2 Energy of the two-dimensional magnetic polaron as a function of the quantum well width in $\mathrm{CdTe} / \mathrm{Cd}_{0.74} \mathrm{Mn}_{0.26} \mathrm{Te} \mathrm{SQW}$ structures [6]. Experimental data are shown by the closed circles and results of a model calculation are presented by the solid line.

\section{Magnetic Polarons in Quantum Wells with Semimagnetic Barriers}

$\mathrm{CdTe} / \mathrm{Cd}_{1-\mathrm{x}} \mathrm{Mn}_{\mathrm{x}} \mathrm{Te}$ single quantum well structures were prepared by molecular-beam epitaxy on (100)-oriented CdTe substrates. The QW structures were grown on a $0.6 \mu \mathrm{m}$ thick buffer layer of $\mathrm{Cd}_{1-\mathrm{x}}$ $\mathrm{Mn}_{\mathrm{x}} \mathrm{Te}$ and contain a set of SQWs that are separated by $1000-\AA$-thick $\mathrm{Cd}_{1-\mathrm{x}} \mathrm{Mn}_{\mathrm{x}} \mathrm{Te}$ barrier layers [9]. Quantum wells with CdTe layer thickness $L_{z}$ ranged from 6 to $300 \AA$ and Mn content in the barrier layers from 0.22 to 0.33 were studied at temperatures from 1.6 to $300 \mathrm{~K}$ and in magnetic fields up to 7.5 Tesla. At low temperatures the photoluminescence (PL) is dominated by a recombination of heavy-hole excitons localized on monolayer fluctuations of $\mathrm{QW}$ width [9]. The exciton magnetic polarons have been studied by use of a technique of selective excitation of localized excitons $[6,10]$.

Fig. 1 displays typical photoluminescence spectra taken from a $12-\AA$-thick SQW under various excitation conditions. A wide PL band (dashed line) is observed under excitation above the $n=1$ heavy-hole exciton energy shown by open arrow. Under selective excitation of localized excitons the PL spectra shown by solid and dotted curves reveal narrower lines well-separated from the excitation energies. The Stokes shift of the PL maximum from the excitation energy $\Delta E=13 \mathrm{meV}$ appears to be independent of the energy of selective excitation within the band of localized states. The Stokes shift is totaly suppressed by an application of external magnetic fields or by a temperature increase up to $30 \mathrm{~K}$ [6]. That is characteristic for the magnetic polaron suppression in $\mathrm{Cd}_{1-\mathrm{x}} \mathrm{Mn}_{\mathrm{x}} \mathrm{Te}$ in the presence of magnetic fields due to the alignment of $\mathrm{Mn}$ spins and by a temperature increase which leads to a decrease of the magnetic susceptibility $[11,12]$. We interpret the shift $\Delta E$ as a result of a two-dimensional exciton magnetic polaron (2D EMP) formation in QWs. Being resonantly excited in the localized state the exciton induces a sizable magnetization within that part of its wavefunction which penetrates to the $\mathrm{Mn}$-rich barrier regions and decreases its energy due to an additional gain in the exciton-ion exchange energy.

The energy of 2D EMP being controlled by the penetration of the exciton wavefunction into the barrier layers is very sensitive to structure parameters namely to the thickness of $Q W$. We have found the 
effective magnetic polaron formation in QWs thinner than $30 \AA$ only. The energy of 2D EMP as a function of the quantum well width is shown by circles in Fig.2. The MP energy is $25 \mathrm{meV}$ in 6- $\AA$-thick QWs and monotonically decreases for thicker QWs. Results of the theoretical consideration of the magnetic polaron formation in QWs done by a variational method in the adiabatic approximation $[7,8]$ are shown by a solid line in Fig.2. They are in a good qualitative agreement with the experimental data. The theoretical model considers the magnetic polaron fomation to be accompanied by the squeezing of the exciton wavefunction in the plane of QW and highlights the importance of the primary exciton localization on the fluctuations of the QW width. The size of the initial exciton localization determined as a fitting parameter has been found to exceed the size of the polaron ground state by a factor of 2-3 [13]. That let us consider the twodimensional magnetic polarons as quasi-free magnetic polarons.

2D EMPs are formed mainly due to the exchange of hole spins with the spins of the Mn ions, because the exchange constant for holes is 4 times larger than that for electrons [14]. In a single quantum well the hole is subject to a net potential well consisting of two contributions of comparable values: the Coulomb potential formed by a strongly confined electron and the valence band offset $[15,16]$. In this case the hole is located in the center of the QW and is not drawn into the barriers in the process of the MP formation. The Coulomb potential is absent in short-period superlattices with wide electron minibands. The experimental studies of the MP formation in superlattices will be presented in section 5 .

Fig.3 demonstrates the anisotropy of the 2D EMP suppression in magnetic fields applied parallel and perpendicular to the structure axis $z$. The anisotropic polaron behaviour which demonstrates the twodimensional character of polarons in QWs, has been found experimentally (circles) and shown by the model calculation (solid lines). The magnetic moments of $2 \mathrm{D}$ heavy-hole excitons are aligned along the $z$-axis owing to the light- and heavy-hole splitting in the valence band resultant from strain and quantum confined effect. As a consequence the magnetic polarons formed from $2 \mathrm{D}$ excitons have their magnetic moments oriented along the $z$-axis. Parallel magnetic fields do not change the alignment of the Mn spins inside the polaron, it aligns the external spins only and this is enough for the MP suppression. To suppress the MP in perpendicular fields the magnetic field must re-orient the spins inside the polaron. In this case the magnetic field behaviour of the MP is a result of a competition between the exchange interactions of the Mn spins with the external magnetic field and with the exciton magnetic moment. This competition results in the threshold like dependence of the 2D EMP energy on perpendicular magnetic field.

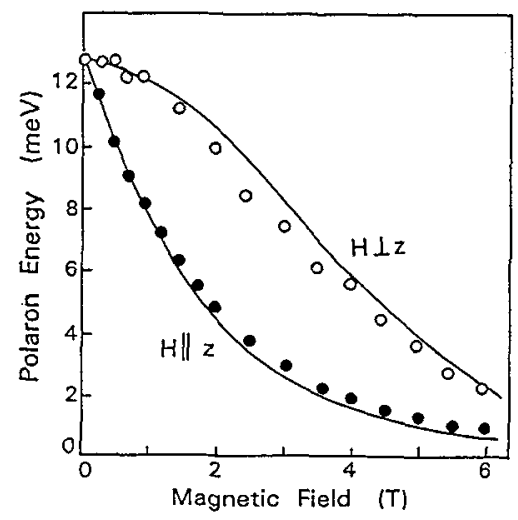

Fig.3 Magnetic polaron energy in a $12-\AA$-thick $\mathrm{CdTe} / \mathrm{Cd}_{0.73} \mathrm{Mn}_{0.27} \mathrm{Te}$ SQW for external magnetic fields parallel (closed circles) and perpendicular (open circles) to the $\mathrm{QW}$ axis at $T=1.6 \mathrm{~K}$. Results of the model calculations are shown by the solid curves [13]. 


\section{Dynamics of 2D Magnetic Polaron Formation}

The dynamics of the 2D magnetic polaron formation has been studied by use of picosecond timeresolved photoluminescence [17]. The evolution of the shift $\Delta E$ after a 5-ps excitation pulse has been measured in 6 and $15-\AA$-thick $\mathrm{CdTe} / \mathrm{Cd}_{0.78} \mathrm{Mn}_{0.22} \mathrm{Te} \mathrm{SQWs}$ at $1.6 \mathrm{~K}$. Fig.4 shows the shifts of the MP line peaks $\Delta E$ as a function of time. The fast initial shift with a saturation at longer delay is characteristic for both QWs. The determined polaron formation times are $100 \mathrm{ps}$ and $250 \mathrm{ps}$ for $6-\AA$ and $15-\AA$-thick QWs, respectively. In thicker QWs the exciton wavefunctions penetrate less into the semimagnetic barriers and a smaller number of Mn spins are involved in the MP formation. This results in the strong dependence of the MP formation time on the QW thickness found experimentally (closed circles in Fig.5) and calculated by solving the time-dependent Schroedinger equation (dashed line in Fig.5) [for details of calculation see Ref.13].
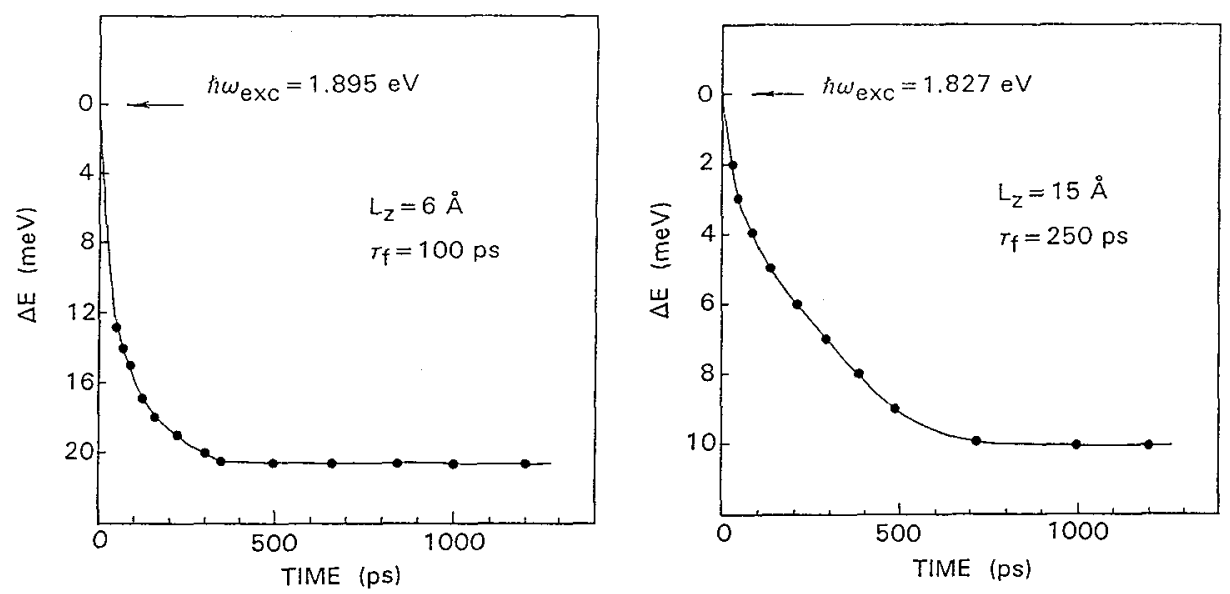

Fig.4 The polaron shift $\Delta E$ as a function of time for $6-\AA$ and $15-\AA$-thick $\mathrm{CdTe} / \mathrm{Cd}_{0.78} \mathrm{Mn}_{0.22} \mathrm{Te} \mathrm{SQWs}$. Resonance excitation energies are shown by arrows. $T=1.6 \mathrm{~K}$. [17]

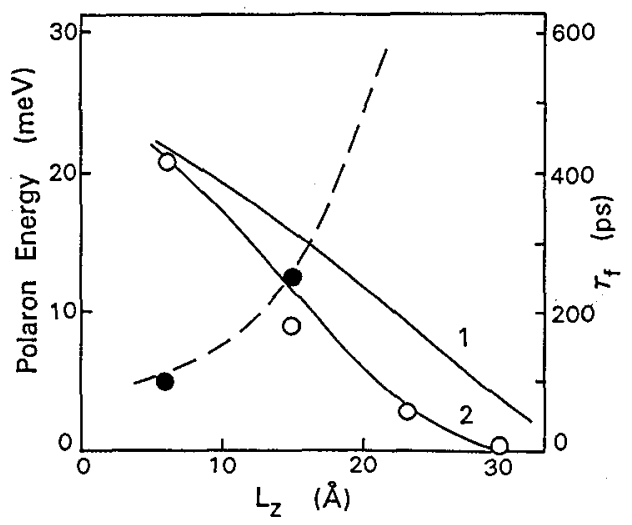

Fig.5 Energy of 2D magnetic polarons (open circles) and the polaron formation time (closed circles) versus quantum well width in $\mathrm{CdTe} / \mathrm{Cd}_{0.78} \mathrm{Mn}_{0.22} \mathrm{Te} \mathrm{SQW}$ structures. $T=1.6 \mathrm{~K}$. Results of the polaron energy calculations are shown by the solid curves for finite (2) and infinite (1) polaron lifetime. Calculated polaron formation time is presented by the dashed curve. [13] 
The saturation value of $\Delta E$ taken at longer delays corresponds to the equilibrium energy of magnetic polarons. In a $6-\AA$-thick SQW the polaron formation time $\tau_{\digamma}=100$ ps is shorter than the polaron lifetime $\tau_{l}=200 \mathrm{ps}$ and the MP energy measured under $\mathrm{cw}$ excitation corresponds to the equilibrium polaron energy. This is not the case for a $15-\AA$-thick SQW where $\tau_{f}=250 \mathrm{ps}$ is longer than $\tau_{l}=170 \mathrm{ps}$ and magnetic polaron does not reach the equilibrium state during its lifetime. In Fig. 5 the equilibrium polaron energy calculated at time $t=\infty$ is shown by the solid curve 1 . The curve 2 demonstrates the polaron energy at $\mathrm{t}=\tau_{l}$ and is in better agreement with experimental data measured under $\mathrm{cw}$ excitation (open circles). A pronounced difference between curves 1 and 2 appears when $\tau_{f}$ exceeds $\tau_{l}$. This means that in QWs thicker than $10 \AA \mathrm{MPs}$ do not reach their equilibrium state during their lifetimes.

The strong decrease of the 2D EMP energy with increasing $L_{z}$ found under $\mathrm{cw}$ excitation is caused by two reasons: (i) the decrease of the equilibrium MP energy and (ii) changes in the dynamics of the MP formation, i.e. increase of the ratio $\tau_{f} / \tau_{l}$. From the results presented in Fig. 5 we conclude that the first reason is the principal one.

\section{Magnetic Polaron as a Probe of Interface Modification of Spin-Glass Phase}

In semimagnetic semicondutor $\mathrm{Cd}_{1-\mathrm{x}} \mathrm{Mn}_{\mathrm{x}} \mathrm{Te}$ the neighboring $\mathrm{Mn}$ ions interact with each other via exchange interaction. At low temperatures and sufficient concentrations of $\mathrm{Mn}$ ions (for $T=1.6 \mathrm{~K} \quad x>0.15$ ) a transition from a paraphase of the magnetic structure to a spin-glass phase takes place [18]. The nearestneighbor exchange interaction of $\mathrm{Mn}$ spins is antiferromagnetic. As a consequence the antiferromagnetically coupled spin pairs and high-ordered clusters give no input to the low field magnetic susceptibility [19] and are considerably less effective in the magnetic polaron formation. At Mn concentrations higher than 0.20 the number of pairs and clusters strongly increases for larger $x$-values. This causes a decrease in the magnetic susceptibility, as shown in Fig. 6 by the dashed line, and a constancy of the MP energy in bulk $\mathrm{Cd}_{1-\mathrm{x}} \mathrm{Mn}_{\mathrm{x}} \mathrm{Te}$ in the $\mathrm{x}$-value range from 0.25 to $0.40[4,5]$ (see Fig.6).
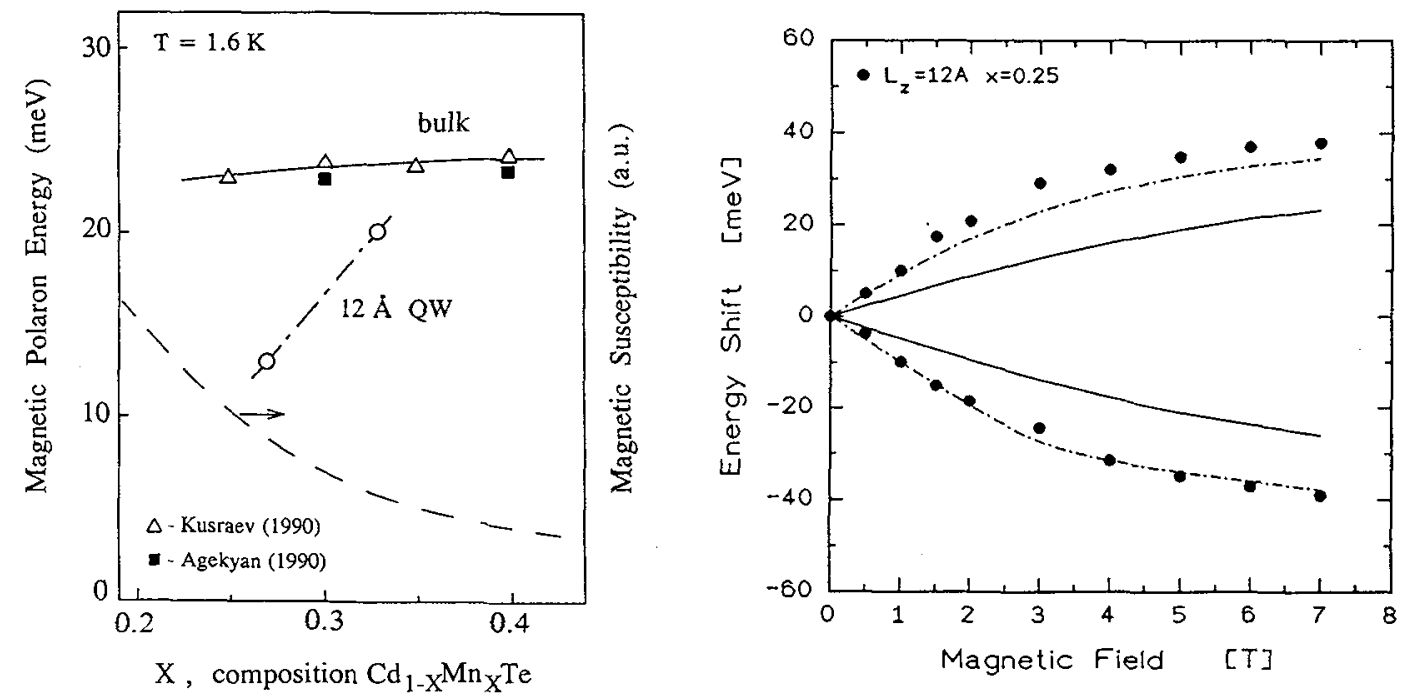

Fig.6 Energies of the exciton magnetic polarons as a function of $\mathrm{x}$-value in bulk $\mathrm{Cd}_{1-\mathrm{x}} \mathrm{Mn}_{\mathrm{x}} \mathrm{Te}$ (triangles [5] and squares [4]) and in 12- $\AA$-thick single quantum wells (circles) [20]. The magnetic susceptibility of $\mathrm{Cd}_{1-\mathrm{x}} \mathrm{Mn} n_{\mathrm{x}} \mathrm{Te}$ is shown by the dashed line.

Fig.7 Zeeman splitting of exciton measured in a $12-\AA$-thick $\mathrm{CdTe} / \mathrm{Cd} d_{0.75} \mathrm{Mn}_{0.25} \mathrm{Te}$ single quantum well (closed circles). The solid and dashed-dotted lines show the results of numerical calculations with parameters discussed in text. [21] 
The $\mathrm{Cd}_{1-\mathrm{x}} \mathrm{Mn}_{\mathrm{x}} \mathrm{Te}$ band gap increases linearly with $\mathrm{x}$-value, which enchances the exciton confinement in the quantum wells and decreases the penetration of the exciton wavefunction into the barriers. Considering this fact together with the constancy of the polaron energy in bulk $\mathrm{Cd}_{1-\mathrm{x}} \mathrm{Mn}_{\mathrm{x}} \mathrm{Te}$ (i.e. in the barrier layers) one could predict a derease of the 2D EMP energy with an increase of the $x$-value for fixed thickness of QWs. We have found a strong increase of the 2D EMP energy for the Mn content range from 0.22 to 0.33 [20]. The data for $12-\AA$-thick QWs are plotted in Fig.6 by open circles. The polaron energy increases from $13 \mathrm{meV}$ for $\mathrm{x}=0.27$ to $20 \mathrm{meV}$ for $\mathrm{x}=0.33$. We attribute this increase to a modification of the spin-glass phase situated at the heterointerface between semimagnetic and nonmagnetic semiconductors. The modification manifests itself in the stronger paramagnetic-like contribution of the interface Mn spins to the 2D EMP energy and to the Zeeman splitting of exciton states [21].

In Fig. 7 the experimental data of the exciton Zeeman splitting in a 12- $\AA$-thick SQW (closed circles) are plotted together with the results of numerical calculations (for details see [21]). The solid lines show the Zeeman splitting calculated with the magnetic parameters of barrier layers corresponding to the bulk parameters. This splitting is nearly twice smaller than the experimental one. The dashed-dotted lines demonstrate the best fit of the experimental data which requires the choice of the stronger paramagnetic parameters for the description of the QW exciton splitting.

Evidencies for the modification of the spin-glass phase in low dimensional systems have been reported for thin $\mathrm{Cd}_{1-\mathrm{x}} \mathrm{Mn}_{\mathrm{x}} \mathrm{Te}$ [22] and $\mathrm{MnSe}$ [23] layers, but a detailed study of the mechanism responsible for this phenomenon requires further investigations. We suggest the $2 \mathrm{D}$ EMP to use as a probe of the magnetic order at the heterointerfaces between semimagnetic and nonmagnetic semiconductors.

\section{Magnetic Polarons in Short-Period Superlattices}

By use of the selective excitation technique we have studied the magnetic polaron formation in short-period superlattices $\mathrm{CdTe} / \mathrm{Cd}_{0.76} \mathrm{Mn}_{0.24} \mathrm{Te}$ (detailed results will be published in Ref. 24). The superlattices (SL) with equal well and barrier thicknesses and periods of $24 \AA, 48 \AA$ and $60 \AA$ have been grown on CdTe substrates after a $0.6 \mu \mathrm{m}$ buffer layer of $\mathrm{Cd}_{0.76} \mathrm{Mn}_{0.24} \mathrm{Te}$. The Bloch-type of perpendicular transport reported for these SLs [25] demonstrates the high structural quality of the samples.
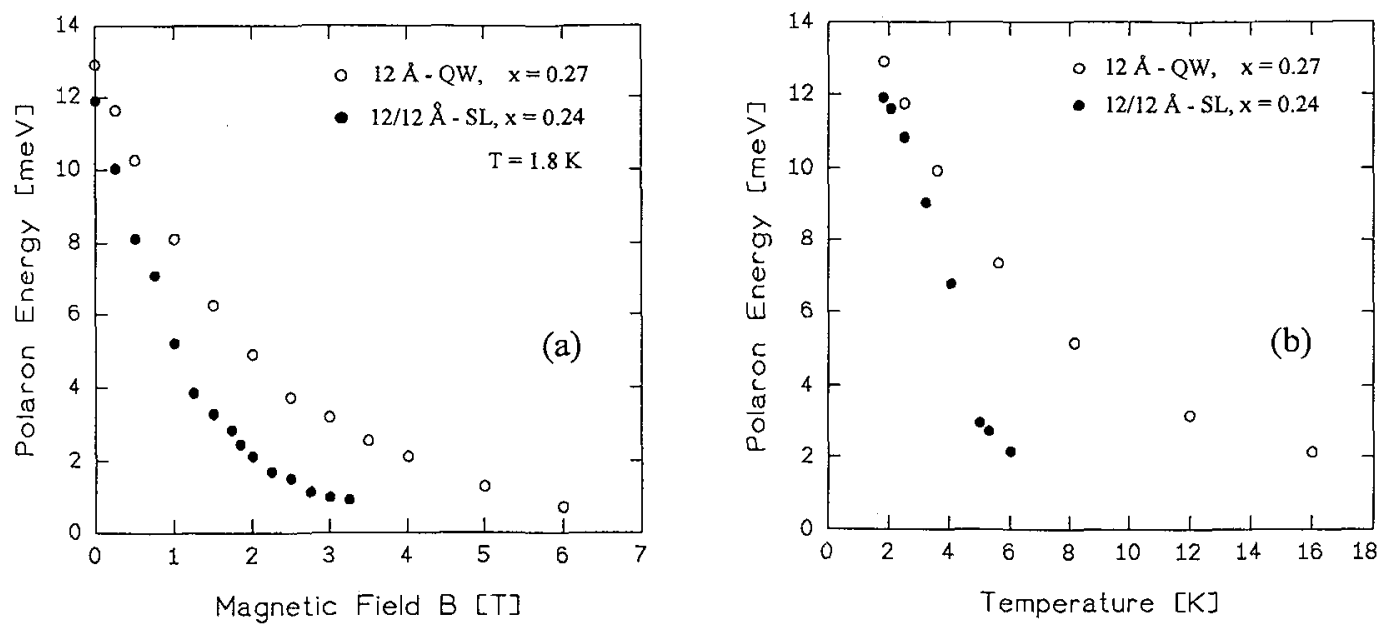

Fig.8 The magnetic polaron energies in a $12-\AA$-thick $\mathrm{CdTe} / \mathrm{Cd}_{0.73} \mathrm{Mn}_{0.27} \mathrm{Te}$ single quantum well (open circles) and in a $24-\AA$-period $\mathrm{CdTe} / \mathrm{Cd}{ }_{0.76} \mathrm{Mn}_{0.24} \mathrm{Te}$ superlattice (closed circles) as a function of external magnetic field (a) and temperature (b) [24]. 
We have measured under selective excitation the well pronounced Stokes shifts of $12 \mathrm{meV}$ in a 24$\AA$-period SL and of $3 \mathrm{meV}$ in a 48 - $\AA$-period SL. No measurable polaron shift has been found in a $60-\AA$ period SL. These energies are very close to the $2 \mathrm{D}$ EMP energies in $12-\AA$ and $24-\AA$-thick SQWs, respectively (see Fig.2). That lets us conclude that even in SLs, where the Coulomb part of the confined potential for the holes vanishes (see discussion in section 2), the hole wavefunctions are not drawn into the barriers in the process of the MP formation.

Fig. 8 shows the magnetic field (a) and temperature (b) polaron suppressions in a $24-\AA$-period SL in comparison with a $12-\AA \AA$-thick SQW with similar barrier content. The magnetic polaron energies in these structures differ slightly at zero magnetic field and temperature of $1.6 \mathrm{~K}$. But the suppression of the SL magnetic polarons is much faster than suppression of the QW polarons both in the external magnetic fields and with the temperature increase. This fact just reflects the stronger paramagnetic properties of Mn spins participating in the magnetic polaron formation in the short-period SLs in comparison with SQW structures. In the $24-\AA$-period SL the barrier layers are 12 - $\AA$-wide, i.e. 5 monolayers only. That means that the majority of the Mn ions is located in the interface or next to the interface layers. The high-ordered clusters, which provide the hardness of the magnetic properties in the spin-glass phase, are also restricted in their size by the barrier thickness. As a result the modification of the spin-glass phase in quasi-twodimensional barrier layers of SLs is even stronger than at the heterointerfaces of SQWs.

\section{Conclusions}

We review the experimental and theoretical investigations of static and dynamical properties of the exciton magnetic polarons in semimagnetic quantum wells and superlattices. The molecular-beam epitaxy growth of the semimagnetic heterostructures gives wide opportunities for controllable changes of the magnetic polaron parameters, such as the value of exchange energy and structure of the wavefunction. The overlap of the exciton wavefunction with semimagnetic barriers, the conditions of the primary exciton localization and the modification of the spin-glass phase at interfaces have a key importance for the magnetic polaron formation in heterostructures

\section{Acknowledgements}

I am indebted to A.Waag for providing the high quality quantum well and superlattice samples. I would like to thank A.V.Kavokin, K.V.Kavokin, G.Mackh, B.Kuhn-Heinrich, W.Ossau, I.N.Uraltsev, G.Landwehr, A.Pohlmann and E.O.Göbel for fruitfull collaboration, as well as I.A.Merkulov and Al.L.Efros for helpful discussions.

\section{Referencies}

[1] Furdyna J.K., J.Appl.Phys. 64 (1988) R29.

[2] Wolff P.A., Semiconductors and Semimetals Vol.25, ed by Furdyna J.K. and Kossut J. (Academic Press, London 1988), p.413.

[3] Golnik A., Ginter J. and Gaj J., J.Phys.C 16 (1983) 6070.

[4] Agekyan V.F. and Serov A.Yu., Soviet Phys. Solid State 32 (1990) 1951.

[5] Kusrayev Yu.G. and Zakharchenya, Proc. 20th Int.Conf. on Physics of Semicond., Thessaloniki 1990, p.738.

[6] Yakovlev D.R., Ossau W., Landwehr G., Bicknell-Tassius R.N., Waag A., Schmeusser S and Uraltsev I.N., Solid State Commun. 82 (1992) 29.

[7] Kavokin A.V. and Kavokin K.V., Soviet Phys. Semicond. 25 (1991) 1053.

[8] Kavokin A.V. and Kavokin K.V., Semicond.Sci.Technol. 8 (1993) 191.

[9] Waag A., Schmeusser S., Bicknell-Tassius R.N., Yakovlev D.R., Ossau W., Landwehr G. and Uraltsev I.N., Appl.Phys.Lett. 59 (1991) 2995.

[10] Yakovlev D.R., Ossau W., Landwehr G., Bicknell-Tassius R.N., Waag A. and Uraltsev I.N., Solid State Commun. 76 (1990) 325. 
[11] Golnik A., Gaj J.A., Nawrocki M., Planel R. and Benoit a la Guillaume C., Proc. 15th Int.Conf. on Physics of Semicond., Kyoto 1980, J.Phys.Soc.Jpn. 49, Suppl. A (1980) 819.

[12] Warnock J., Kershaw R.N., Ridgley D., Dwight K., Wold A. and Galazka R.R., Solid State Commun. 54 (1985) 215.

[13] Yakovlev D.R., Ossau W., Waag A., Bicknell-Tassius R.N., Landwehr G., Kavokin K.V., Kavokin A.V., Uraltsev I.N. and Pohlmann A., Proc. 21 st Int.Conf. on Physics of Semicond., Beijing 1992, p. 1136.

[14] Gaj J.A., Planel R. and Fishman G., Solid State Commun. 29 (1979) 435.

[15] Chang S.K., Nurmikko A.V., Wu J.W., Kolodziejski L.A. and Gunshor R.L., Phys.Rev.B 37 (1988) 1191.

[16] Efros Al.L., Soviet Phys. Semicond. 20 (1986) 808.

[17] Yakovlev D.R., Ossau W., Landwehr G., Bicknell-Tassius R.N., Waag A., Schmeusser S. Uraltsev I.N., Pohlmann A. and Göbel E.O., J.Crystal Growth 117 (1992) 854.

[18] Furdyna J.K. and Samarth N., J.Appl.Phys. 61 (1987) 3526.

[19] Oseroff S.B., Calvo R., Giriat W. and Fisk Z., Solid State Commun. 35 (1980) 539.

[20] Yakovlev D.R., Festkörperprobleme / Advances in Solid State Physics, Vol.32, ed.by U.Rössler (Vieweg, Braunschweig 1992), p.251.

[21] Ossau W.J. and Kuhn-Heinrich B., Physica B 184 (1993) 422.

[22] Awschalom D.D., Hong J.M., Chang L.L. and Grinstein G., Phys.Rev.Lett. 59 (1987) 1733.

[23] Chang S.K., Lee D., Nakata H., Nurmikko A.V., Kolodziejski L.A. and Gunshor R.L., J.Appl.Phys. 62 (1987) 4835.

[24] Mackh G., Yakovlev D.R., Ossau W., Waag A. and Landwehr G., to be published.

[25] Hellmann R., Pohlmann A., Yakovlev D.R., Waag A., Bicknell-Tassius R.N. and Landwehr G., Proc. 21st Int.Conf. on Physics of Semicond., Beijing 1992, p.1008. 